\title{
Literatura fractal
}

\section{Gilberto Antonio Nava Rosales*}

Resumen: La literatura fractal se ha presentado como una construcción que implica dependencia, sin embargo ésta no requiere dicha estructura, para la comprobación de esta hipótesis se propone el análisis de dos cuentos de Julio Cortázar: "Orientación de los gatos" y "Continuidad de los parques" donde se localizan algunos elementos enunciados por Mandelbrot que definen a un fractal matemático.

Palabras clave: literatura, fractal, Cortázar, cuento corto.

Abstract: The fractal literature has been showed as a costruction that needs other texts, however this literature does not need them, in order to check that hypothesis, it is proposed the analysis of two stories of Julio Cortázar: "Orientation ofCats"and"Continuityofparks",wheretherearelocatedsome elements enunciated by Mandelbrot that define a mathematical fractal.

Key words: literature, fractal, Julio Cortázar, short story.

INTRODUCCIÓN

La belleza en las obras de arte es un tema recurrente durante todas las épocas, aunque hay diversas propuestas respecto a sus características, una gran parte de las ideologías planteadas contienen elementos similares al enumerar los rasgos para describirla. Una propiedad importante es la proporción armónica; desde la antigüedad, tanto filósofos como matemáticos han tratado de fundamentar

* Estudiante de la Licenciatura en Lengua y Literatura Hispánicas en la Facultad de Filosofía y Letras de la Universidad Nacional Autónoma de México. 
dicha tesis.' De ese modo surge "la divina proporción", canon que aparece como hipótesis de la relación longitudinal entre las partes de un todo. ${ }^{2}$ Así, "la sección áurea" ha dado pauta para la formación y estudio de los fractales: ${ }^{3}$

Mandelbrot denominó fractales (del latín fractus, irregular) al conjunto de formas que, generados normalmente por un proceso de repetición, se caracterizan por poseer detalle a toda escala, por tener longitud infinita, por no ser diferenciables y por exhibir dimensión fraccional. (Talanquer, 2009: 25).

Es decir que un fractal se forma básicamente por un principio de repetición, en él es posible observar la misma imagen desde cualquier ángulo o acercamiento. Dicho suceso parece innegable en distintos ámbitos, como la ubicación de las galaxias, las fluctuaciones en las bolsas de valores, incluso, en la repetición de letras en un texto (11). En teoría, así como el universo parecía estar regido por el "número dorado", lo fractal aparece como el factor presente en toda la naturaleza. Mas no todo en la natura lo es (al menos no en esta realidad):

Si toda sucesión de eventos en el tiempo tuviera estructura fractal, nuestra vida podría ser un verdadero infierno. Cada instante contendría todo pasado y futuro y viviríamos constantemente nuestra propia muerte, pero esto es exagerar. Si toda distribución de materia en el espacio siguiera las reglas de la geometría fractal, estaríamos en todas partes, seríamos el universo entero. (92)

Por otra parte, existen universos ficticios donde el tiempo junto con otros muchos de sus componentes pueden adquirir la propiedad fractal. Se pueden localizar en la literatura, id est la literatura fractal. Alberto Viñuela la define haciendo énfasis en los elementos recursivos tales como las "tautologías", "historias cíclicas" y "cajas chinas"4 (otro elemento que acota es la reduplicación ${ }^{5}$ ).

1 Para una explicación más amplia vease La Divina Proporción de Juan Ignacio Extremiana Aldana.

2 Fra Luca Paccioli lo propuso en 1509 con su tratado De Divina Proportione. En él argumentaba que era posible encontrar un número como resultado constante al dividir el lado mayor de un rectángulo entre el lado menor del mismo. Una prueba puede realizarse en aquellos utilizados por los griegos para su arquitectura.

3 Aparentemente "el número dorado" y los fractales son equiparables; sin embargo, hay una distinción significativa: mientras que el número de Paccioli se desarrolla en el conjunto de los números reales, los fractales pueden formarse en cualquier conjunto numérico —reales y complejos (llamados erróneamente imaginarios). Podría decirse que "la divina proporción" es el principio de la geometría fractal.

4 "Llamo literatura fractal a todo aquel escrito que manifiesta propiedades similares a las de los objetos fractales, centrándose sobre todo en los elementos recursivos, es decir, que hacen referencia a sí mismos [...] incluiré aquellas que traten el tema de la recursividad o que recojan la idea de fractal y hayan sido escritas antes de que éstos se definieran de forma matemática" (Viñuela, 2001).

5 "Fenómeno [...] mediante el cual se designa en muchas lenguas una intensificación o un aumento cuantitativo o dimensional" (Simone, 2001: 60). 
Paniagua (2007) retoma dicha definición, luego la limita al nivel sintáctico de las oraciones, ${ }^{6}$ pero al hacerlo se abandonan diversos aspectos literarios, como la invención de lenguajes ${ }^{7}$ — por ejemplo, el glíglico en Rayuela (Cortázar, 2006: 488) - o el uso de "palabras virtuales"8 para dar el efecto fractal al texto. Lauro Zavala también hace uso del término "fractal"; menciona que es una característica de la minificción, ${ }^{9}$ así como un texto perteneciente a una serie, el cual puede ser analizado como un texto independiente o junto con la totalidad, pues mantiene una relación por su temática o estilo. ${ }^{10}$ Asevera la diferencia entre fragmento y fractal con la autonomía del primero y la dependencia del segundo a una serie (Zavala, 2006: 135); de lo anterior desprende su certeza: "El detalle o fractal es una unidad narrativa que sólo tiene sentido en relación con la serie a la que pertenece" (135). Si se verifica el concepto literal, el fragmento y el fractal, en efecto se oponen; sin embargo, éste último no necesita de ningún otro componente para adquirir sentido. Un texto fractal no requiere forzosamente estar subordinado a una serie narrativa de textos integrados: ${ }^{11}$ sólo los signos contenidos, pueden crear la fractalidad por sí mismos.

En resumen, Viñuela se aproxima a la traslación del concepto "fractal" al ámbito literario, pues respeta la metodología básica que Mandelbrot propuso, para someterla a algunas reglas narrativas y lingüísticas. Quizá sólo omitió la especificación del resto de los recursos literarios para crear un fractal, así como una taxonomía específica del tipo de fractal literario que se encuentra. Por lo tanto,

6 "Está claro que a una oración, compuesta por un sistema de signos con sus significados y significantes, no se la puede someter a la secuencia de un algoritmo fractal, pues perdería su coherencia sintáctica; pero lo que sí se puede hacer es imitar los modelos fractales, respetando la sintaxis, para generar oraciones y textos que conformarán lo que se puede denominar como literatura fractal" (Paniagua, 2007).

7 Viñuela también refiere a lenguajes fractales en su artículo.

8 "[...] secuencias fonémicas que podrían (si existieran) pertenecer a la misma lengua, pero no están actualmente aprovechadas" (Simone, 2001: 98). Cabe mencionar que las combinaciones de morfemas también pueden ser palabras virtuales.

9 Lo mencionó en su ponencia del Segundo Encuentro de Minificción titulada "De la teoría de la novela a la teoría de la minificción". Llevado a cabo el 10 de marzo del 2010.

10 “.... Considerar a un texto como fragmentario, o bien considerar que un texto puede ser leído de manera independiente de la unidad que lo contiene (como fractal de un universo autónomo) es uno de los elementos penalizados por la lógica surgida en la llustración" (Zavala, 2004: 77). Luego indica: "la idea de que un fragmento no es un detalle, sino un elemento que contiene una totalidad que merece ser descubierta y explorada por su cuenta" (80). Después en "Una conclusión para empezar: fragmentos, detalles y fractales" define al detalle como: "segmentación provisional de una unidad global íntegra e indivisible" (Zavala, 2006: 135). Para después reducir el fractal a "un tipo particular de detalle", pues "contiene rasgos genéricos, estilísticos o temáticos que comparte con los otros de la misma serie" (135).

11 “Textos que se enlazan entre sí, conformando un patrón de relación ... repetición de personajes, ambientación geográfica, [etcétera]" (Brescia, 2006: 7-8). 
un texto fractal debe ser aquel que sin depender de una serie o ciclo cuentístico, con base en la repetición de signos lingüísticos, no sólo en el orden sintáctico sino también en el sintagmático, fonológico, morfémico, semántico, semiótico, y mediante las leyes de una gramática preestablecida, pueda formar algún texto narrativo o poético donde sea posible observar una totalidad en cada una de las partes, de uno o más niveles de los antes mencionados. ${ }^{12}$ La definición parece englobar sólo novelas, pero es posible aplicarla a textos de menor extensión, como los cuentos, en este caso: "Orientación de los gatos” (Cortázar, 1999: 13-18) y"Continuidad de los parques" (Cortázar, 1995: 14-15).

\section{Fractales en dos cuentos de Julio Cortázar}

Así, se propone el siguiente análisis de dos cuentos para demostrar la existencia de "lo fractal" en ellos; con la definición de "literatura fractal" del apartado anterior.

\section{Mujer fractal}

El cuento "Orientación de los gatos" presenta la historia de un triángulo relacional de una pareja con su gato: Osiris-Alana-Narrador. En la narración se mencionan las distintas aproximaciones fallidas del narrador por alcanzar a su mujer (Alana), quien parece entenderse más con la mascota.

Uno de los aspectos que se marcan es la mímesis mujer-gato:"la mujer protagonista es asimilada, por su conducta misteriosa, a Osiris, el gato" (Gambetta, 2007: 83); de hecho en "la imaginería nietzscheana el gato es una mujer. En "Orientación de los gatos", el proceso actancial de Alana es un lento pasaje desde el lado de allá hasta el lado de acá para ingresar en la órbita de Osiris" (Mora, 2000: 107). Lo anterior habla de una relación Alana-Osiris en la cual, el narrador no puede penetrar, debido a que "plenamente consciente de las dos realidades, califica de 'infranqueable' distancia que lo separa de su realidad y la de Alana" (Payán, 2009: 4).

Otros aspectos remarcables son la existencia de "una metafísica de la mirada que comprende desde la simple contemplación hasta una mirada creativa, pasando por la posesión amorosa" (Mora, 2000: 102) y la vinculación con la pintura: el cuento

12 Ésto es necesario si lo que se pretende es trasladar el concepto de fractal a la literatura y puesto que no todos los fractales matemáticos son completamente autosimilares. Hay fractales lineales, que mantienen la autosimilitud en todos los puntos; fractales no lineales, en donde a cada nivel de aproximación aparecen rasgos peculiares que hacen perder la autosimilitud; fractales aleatorios, cuya construcción está constituida por elementos condicionados al azar (Talanquer, 2009: 37-61). 
"es el caso más palpable de la interrelación entre plástica y literatura, pues el narrador observa en primera persona cómo su enigmática amada Alana desaparece dentro de un cuadro junto a su no menos críptico gato Osiris" (Calzado); del mismo modo, la "metafísica de miradas" pide que "se recurra al arte pictórico por excelencia, la espacialidad, estrechamente relacionada con el orden de la visión y la perspectiva" (Mora, 2000: 102).

Sin embargo, se deja (u obvia) el papel del narrador. El fractal surge a partir de esa "metafísica de la mirada" que propone Carmen de Mora: "Verbos, sustantivos y adjetivos referentes a la mirada, la vista y los ojos se superponen y suceden en el diorama textual" (102). Mas no son los ojos el símbolo recurrente y omnipresente, sino Alana. Es ella el símbolo lingüístico recurrente durante todo el texto. Si se observa detalladamente, tanto Alana como Osiris aparecen cumpliendo distintas funciones gramaticales en los enunciados hasta abarcar las generales: sujeto, complemento indirecto, objeto directo, complemento circunstancial; siendo ellos, en conjunto o aislados, el núcleo de cada una. Pero Osiris no aparece durante toda la narración, durante el transcurso de ver las pinturas en la galería no está, reaparece solamente al final; por ello podría afirmarse que Alana es omnipresente, ya que cumple con esa propiedad durante todo el relato y en cada parte de los enunciados, cambiando su posición sintáctica o gramatical.

Dicha ubicuidad es perceptible gracias a la focalización del narrador. En varias ocasiones la menciona como elemento autorreferente mediante el uso del pronombre reflexivo "se" o con simples construcciones gramaticales como "la entrevisión de Alana en Alana" (Cortázar, 1999: 14). Las alusiones de la mujer como fractal se observan en varios aspectos.

El uso repetitivo del nombre. El narrador la evoca mayoritariamente con su nombre, con lo cual logra una repetición constante, esto es visible si se comparan las veces que usa un sustitutivo a las veces que usa el sustantivo; por ello aparecen líneas como "la música la desnudaba de una manera diferente, la volvía cada vez más Alana porque Alana no podía ser solamente esa mujer que siempre me había mirado de lleno sin ocultarme nada" (14) durante todo el texto. Así se percibe la posibilidad de ahondar más en ella, de adentrarse en lo que ella es, aunque se ve su imagen, al mirarla desde distintos ángulos aparece como la misma, de hecho parece más ella misma desde cada perspectiva que el narrador recurra para develarla. ${ }^{13}$

Alana es un ser físicamente determinado. "Frente a un cuadro o un grabado Alana se despojaba todavía más de eso que creía ser, por un momento entraba en un mundo imaginario sin salir de sí misma" (14). Nunca deja su dimensión,

13 Recuérdese la propiedad que Mandelbrot afirmó sobre los fractales de presentar la misma imagen desde cualquier ángulo o punto de vista. 
la cual parece estar entre la realidad del relato y las distintas realidades artísticas ante las cuales el narrador la contrapone (la música y la pintura), es decir una especie de dimensión fraccional, que el narrador confirma: "sus altos y sus memorias se inscribían en un tiempo diferente del mío" (15), esto se hace más notorio al final:

[...] ella había ido al cuadro pero no estaba de vuelta, seguía del lado del gato mirando más allá de la ventana donde nadie podrá ver lo que ellos veían, lo que solamente Alana y Osiris veían cada vez que me miraban de frente (18).

Ya sea que literalmente Alana se mete al cuadro o no, nunca abandona ni pertenece a ambos universos, pues la relación Alana-narrador la une a la realidad que existe dentro del texto, al mismo tiempo que ya se encuentra inmersa en la pintura, por lo tanto está en un limbo dimensional.

Léxica y semánticamente, dentro de la estructura del relato, es un ser múltipleunitario. El uso del sustantivo "Alana" en su forma plural "Alanas" la convierte de inmediato en ese ser polisémico: "la música me dejó entrever otras Alanas" (14). En cuanto al significado, la multiplicidad es más explícita: "el cromatismo interior que la recorría hasta mostrarla otra, allí donde la otra era siempre Alana sumándose a Alana" (16). También aparece la figura geométrica del triángulo multiplicado, que al combinarse con Alana le da el matiz fractal; todo esto debido a la percepción del narrador ("la metafísica de miradas"):

[...] mis ojos multiplicaban un triángulo fulminante que se tendía de ella al cuadro y del cuadro a mí mismo para volver a ella y aprehender el cambio, la aureola diferente que la envolvía un momento para ceder después a un aura nueva, a la tonalidad que la exponía a la verdadera, a la última desnudez. Imposible prever hasta dónde se repetiría esa ósmosis, cuántas nuevas Alanas me llevarían por fin a la síntesis de la que saldríamos los dos colmados... (16).

Los distintos cambios que sufre a través del tiempo del relato la confirman como un ser múltiple que al mismo tiempo es uno. Estas metamorfosis, como ya se mencionó, se verifican en los distintos aspectos bajo los cuales el narrador la observa. Aquel proceso acontece, pues él trata de acercarse a ella, "diferenciarla": "Contra Alana, más allá de Alana yo la buscaba para amarla mejor..." (14).

La búsqueda define el nivel actancial del protagonista narrador... Postula Sartré en El Ser y la Nada que amar es querer alcanzar al otro en su propia libertad, no sólo para conquistar su cuerpo, sino para dominar esa misma subjetividad que al mirarnos nos domina, pero esa búsqueda es en sí inalcanzable: "el otro es por principio lo inaprensible: me escapa cuando lo busco y me posee cuando escapo de él". La narración avanza hasta aquí desde un comienzo en que el protagonista se siente mirado, observado, hasta lo que, en términos sartreanos, sería la liberación de esta esclavitud 
de la mirada del otro que lo convierte en objeto. Conocer a Alana con la mirada es una aspiración que se desea liberación... el protagonista de "Orientación de los gatos" mira a Alana para poseerla en plenitud, un deseo que se sabe destinado a fracasar, pero que no cesa de ser deseo (Mora, 2000: 104).

En efecto, mediante la búsqueda de Alana, con la cual se pretende la liberación - puesto que desde el principio el narrador está atrapado en ella: "porque eso también era yo, eso era mi proyecto Alana, mi vida Alana" (14) — basada en la aprehensión, ocurren todas las transformaciones que el narrador admira como la ruta que poco a poco le guía hacia ella:

Pero ahora mi esperanza empezaba a cumplirse casi insoportablemente, desde nuestra llegada Alana se había dado a las pinturas con una atroz inocencia de camaleón, pasando de un estado a otro sin saber que un espectador agazapado acechaba en su actitud, en la inclinación de su cabeza, en el movimiento de sus manos, el cromatismo interior que la recorría hasta mostrarla otra, allí donde la otra era siempre Alana sumándose a Alana, las cartas agolpándose hasta completar la baraja. A su lado, avanzando poco a poco a lo largo de los muros de la galería, la iba viendo darse a cada pintura... yo sabiendo que mi larga búsqueda había llegado a puerto y que mi amor abarcaría desde ahora lo visible y lo invisible, aceptaría la limpia mirada de Alana sin incertidumbres de puertas cerradas, de pasajes vedados (16).

El fracaso ocurre porque ella es inasible. Tal propiedad la adquiere al ser delimitada e infinita. Por principio se sabe que detrás de sus ojos azules existe otra: "Detrás de esos ojos azules hay más, en el fondo de las palabras los gemidos y los silencios alienta otro reino, respira otra Alana" (13-14). La infinitud se indica desde el párrafo anterior con esa especie de contraposición de espejos en las miradas confrontadas de los dos protagonistas:

Alana es mi mujer y la distancia entre nosotros es otra, algo que ella no parece sentir, pero que se interpone en mi felicidad cuando Alana me mira, cuando me mira de frente igual que Osiris y me sonríe o me habla sin la menor reserva, dándose en cada gesto y cada cosa como se da en el amor, allí donde todo su cuerpo es como sus ojos, una entrega absoluta, una reciprocidad ininterrumpida. (13)

Incluso, por un instante parece comprensible, corpórea, pero se le redescubre como un monstruo geométrico. La vez que estuvo más próximo a ella —al final del relato, antes de ver el último cuadro de la galería - es cuando la pierde en absoluto, como si descifrase el patrón del fractal que ella simboliza, sólo para perderla. En los cuadros aparecen distintos símbolos que pretenden apresarla:

Ya no podía extrañarme que esa otra pintura donde una reja de agudas puntas vedaba el acceso a los árboles linderos la hiciera retroceder como buscando un punto de mira, de golpe era la repulsa, el rechazo de un límite inaceptable ... Cada nueva pintura arrasaba a Alana despojándola de su color 
anterior, arrancando de ella las modulaciones de la libertad, del vuelo, de los grandes espacios, afirmando su negativa frente a la noche y a la nada, su ansiedad solar, su casi terrible impulso de ave fénix (16-17).

La negativa a las limitaciones remiten de nuevo a su indeterminación en el plano narrativo. Podría decirse que su instinto la obliga a rehusar las fronteras con que el narrador pretende asirla; es decir, ya sea porque se transforme en fractal o porque sea un fractal, los límites no pueden contenerla, aunque sí predecirla por momentos, pues en las figuras fractales el único límite es la dimensión. ${ }^{14}$ Esa sensación de acorralamiento la empuja a buscar una salida (cualquier salida), que aparece al final de la galería junto con una nueva transformación que "... hizo de ella una lenta estatua nítidamente separada de los demás, de mí que me acercaba indeciso buscándole los ojos perdidos en la tela" (17), ésta "la distingue y separa de las demás personas, del narrador mismo" (Payán, 2009: 4). Ocurre frente al cuadro del gato idéntico a Osiris, allí Alana se desprende totalmente de las ataduras que había logrado hacer el narrador durante el paseo por la galería: "De alguna manera sentí que el triángulo se había roto; cuando Alana volvió hacia mí la cabeza el triángulo ya no existía, ella había ido al cuadro, pero no estaba de vuelta" (18). La fractura del triángulo es la expulsión de Alana del plano del protagonista, su vuelta a esa dimensión indeterminada, a la cual aparentemente sólo el gato tiene acceso.

Todo el relato traza una progresión en que el narrador, fascinado por la imagen de la mujer, la observa, la ve metamorfosearse, despojarse de la energía vital hasta adquirir la solidez mineral de una estatua [lo cual no significa] dejar de ser sino entrar en otro orden oculto a la mirada. Al final, queda de nuevo solo frente a Alana y Osiris que lo miran. La soledad y la fascinación están hermanadas, por ser ésta la mirada "de lo incesante y de lo interminable"... ver sin poder llegar del todo a dominar el objeto (Mora, 2000: 108).

Por lo tanto, la mirada del narrador es lo que descubre a la mujer como ese ser inasible, omnipresente, delimitado por su cuerpo ("la forma que la forma"15), de naturaleza indeterminable; la cual se le escapará siempre.

14 Relativamente, pues la dimensión fraccional puede ser igual a un número irracional, es decir con número infinito de decimales, por lo tanto indeterminado (Talanquer, 2009: 21).

15 Paráfrasis de un fragmento del capítulo 14 de la serie animada japonesa Neon Genesis Evangelion dirigida por Hideki Anno, producida por el estudio Gainax en 1995. Doblaje a cargo de ADV. "La forma que me forma" en el original. 
NARRADOR, EL ORIGEN DEL FRACTAL

“Hay un cuento de Julio Cortázar - 'Continuidad de los parques' - en el que el personaje principal lee en un sillón verde una novela a cuyo final un asesino mata a un hombre que lee en un sillón verde una novela" (Fortea, 2007: 65). Es obvia la recursividad en la trama de este relato; sin embargo, no es tan clara la intervención del narrador en él. Mediante un análisis del narrador es posible observar la imagen del juego de espejos contrapuestos que se reflejan a sí mismos innumerables veces (uno de los fractales más básicos). En primer lugar, el tiempo verbal del relato, que “... está estructurado de tal manera que el lector es el coautor de un texto que no concluye sobre la página" (Zavala, 1993: 124), pues existen demasiados puntos de indeterminación dentro de la historia, específicamente el final, concluye con la imagen de la cabeza de un hombre, que lee una novela, recargada sobre el sillón verde, contrariando la afirmación de que realmente lo mata.

Cortázar no cumple la función de generar una complicidad del lector y narrador, a expensas del autor ... sino que cumple la función de dislocar los niveles narrativos, de contraponerlos unos a otros, de producir ... diversas versiones de un hecho que nunca termina por ser narrado como una totalidad desde un único punto de vista ... el hacer converger diversos planos dentro del relato presenta [a] un narrador que, al haber perdido la noción exacta de su situación y de la realidad misma, recurre involuntariamente a diversas versiones y diversas temporalidades de su propia consciencia, con el fin de lograr ... la impresión de una totalidad y una coherencia que sólo existe en la lectura del relato como un todo, pero no en la memoria fragmentaria del narrador múltiple (141).

Es decir, el narrador de la historia es único. Éste mismo da la visión confusa de los acontecimientos con "la falta de diferenciación entre el sueño (ficción) y la realidad" (Jaeck, 1998: 35). ${ }^{16}$ Indudablemente éste cuento es circular, "la última línea del relato refiere de vuelta a la primera" (6): $:^{17}$ el cuento concluye aparentemente en el mismo momento en que empieza: "En continuidad de los parques... asistimos a otro recurso que llamaremos circularidad y que consiste en una vuelta a la situación anterior, previo paso a un acto de escape de la realidad apoyado en el sueño o la lectura" (Amícola, 1969: 35). Normalmente estos nexos se logran con metaficción. Éste relato parece presentarla. Si se utiliza la teoría de Genette donde considera para el análisis narrativo al tiempo, persona y los niveles narrativos (los elementos propios del relato como enunciado verbal) (113), se observa en

16 La traducción es mía.

17 La traducción es mía. 
este caso que el análisis arroja que el texto "pone en juego dos historias, cada una de las cuales tiene una relativa autonomía sintáctica y se inserta en un universo con reglas diferentes" (115).

Por lo tanto, el narrador nunca cambia, no está enmarcado por un cambio, no existe (Zavala, 1993: 124). No hay una transición de un narrador del relato a un narrador de la novela dentro del relato; es el mismo narrador quien describe superficialmente lo que el personaje lee, este hecho es visible en la omisión del diálogo entre los dos personajes de la novela: “Un diálogo anhelante corría por las páginas como un arrollo de serpientes" (Cortázar, 1995: 14). “La narración del primer narrador intenta fusionarse con la enunciación del narrador de la novela. Pero no se abandona un nivel narrativo para dar lugar a otro" (118). Es decir, el narrador emula ese universo reflejado.

El paso de la realidad a la ficción se observa en el primer párrafo, de un uso concreto del participio y pretérito perfecto:18 "Había empezado a leer una novela", "La abandonó por negocios urgentes", "volvió a abrirla" (13); poco a poco empieza a surgir el pretérito imperfecto en oraciones yuxtapuestas a otras construidas con pretérito perfecto: "se puso a leer los últimos capítulos. Su memoria retenía sin esfuerzo los nombres", "la ilusión novelesca le ganó casi enseguida. Gozaba del placer casi perverso" (13). A partir de la oración "Primero entraba la mujer" (13) el resto de los tiempos verbales (simples o compuestos) tienen como núcleo el pretérito imperfecto. Es decir, indica una acción que continúa hasta el momento de la enunciación, en este caso de la lectura. A causa de ello"el texto que está leyendo el protagonista [...] coincide con el tiempo real del relato" (Ros, 1990: 244) pues "el narrador ha pasado de una historia a otra, pero no de uno a otro nivel narrativo" (Filinich, 1996: 117) por lo tanto ocurre que "el personaje de un primer relato se convierte en lector de otro relato" (115).

El último párrafo del cuento permite pensar en la unificación de realidades, pues "la narración intenta borrar las marcas del acto de lectura para fundirse con la enunciación de las acciones narradas en la novela" (117).

El narrador único que unifica lo fantástico con lo real dentro del relato es el factor que permite la fractalidad del cuento. Implícitamente se dan indicios de lo que ocurrirá; es decir, que la acción del final, el encuentro entre el lector y el

18 “Podríamos caracterizar a este primer narrador según el modo como se manifiesta: se trata de un narrador explícito que alude al protagonista en tercera persona y narra los hechos en tiempo pasado (utilizando, en un primer momento del relato, preferentemente el pretérito indefinido). El uso de la tercera persona verbal connota un cierto distanciamiento de los hechos asegurando la objetividad y verosimilitud a lo narrado... Si bien el narrador extradiegético mantiene plenamente su función narrativa (destina el relato un virtual narrativo y verbaliza la narración), asume la perspectiva del personaje, limitando su conocimiento y alcance de su visión a aquello que el personaje puede tener acceso" (Filinich, 1996: 116). 
asesino, está presente desde el principio del relato, "todo está decidido desde siempre" (Cortázar, 1995: 14). Del mismo modo la imagen del "espejo narrativo" es otro elemento autosimilar, característico de los fractales, que remite no sólo a las acciones del mismo relato sino a la acción de quien lee el cuento; es decir, que el lector lee un cuento acerca de alguien que lee una novela, la cual narra cómo dos personajes pretenden matar a un tercero, a quien uno de ellos encuentra leyendo una novela. Así la inconclusión permite esa imagen, pues detiene al amante con la imagen del hombre leyendo.

\section{CONCLUSIONES}

Los ejemplos mostrados permiten vislumbrar la propiedad fractal dentro de la literatura. Podría decirse que son los contraejemplos a la teoría que propone Zavala al respecto de la fractalidad y la fragmentariedad de los textos narrativos. Esta propiedad también puede difundirse en la poesía. ${ }^{19}$ Si bien es cierto que se puede mirar un objeto el tiempo suficiente para ver en él un patrón definido - como un fractal, la exageración de la teoría-, también lo es el hecho de que estas figuras son inherentes al orden de las cosas; y si se encuentran en la naturaleza o el cosmos, bajo la consideración de que la literatura, como toda obra de ficción, simula la realidad bajo ciertas condiciones determinadas - curioso parecido a la natura real-, ¿por qué no habrían de encontrarse fractales literarios autónomos e independientes?

Zavala afirma que un fractal sólo cobra sentido cuando se le coteja con la serie a la que pertenece; sin embargo, los cuentos "Orientación de los gatos" y "Continuidad de los parques" no requieren de serie o ciclo cuentístico alguno para que el fractal sea inteligible al lector. Por ejemplo, a lo anterior podría añadirse que la repetición del nombre "Alana" en el cuento "Orientación de los gatos" crea un fractal fonológico con base en el fonema /a/ pues es el predominante, ya que aparece tres veces en el nombre de la protagonista y que esta repetición de vocales no sucede en el resto de los cuentos de "Queremos tanto a Glenda". La propiedad fractal no es única de las novelas o de las minificciones en ciclos cuentísticos, también se localiza en cuentos o cuentos breves como los aquí expuestos.

19 Mario Markus, matemático chileno, publicó un libro Poemas de invierno, en donde trata de unificar la ciencia con el arte; otro de los tantos propósitos encomendados a los fractales. 
BiBLIOGRAFÍA

Ainsa, Fernando. (1992) Cristina Peri Rossi: del otro lado de la puerta. pp. 219-227. [en línea]. Recuperado el 21 de marzo del 2010 de http://www.raco.cat/index.php/Scriptura/article/viewFile/94419/142620

Amícola, José. (1969) Sobre Cortázar. Argentina: Escuela.

Brescia, Pablo y Romano Evelia. (2006) Estrategias para leer textos integrados. El ojo en el caleidoscopio. México: UNAM, pp. 7-42.

Cortázar, Julio. (1995) Final del juego. (5a. ed.) México: Alfaguara.

, (1999) Queremos tanto a Glenda. (4a reimp. de la 1a. ed.) México: Alfaguara. , (2006) Rayuela. (2a. ed) España: Punto de lectura.

Extremiana Aldana, J. Ignacio. (2008) La Divina Proporción. [en línea]. Recuperado el 28 de febrero del 2010 de http://www.google.com.mx/url?sa=t\&sourc $\mathrm{e}=$ web\&ct=res\&cd=7\&ved=0CCAQFjAG\&url=http\%3A\%2F\%2Fwww.sectormatematica.cl\%2Farte\%2Fdivina_proporcion.pdf\&rct=j\&q=luca+paccioli+d e+divina+proportione\&ei=zgGKS_n4FYqMswPS36iGAw\&usg=AFQjCNEQ18 08bWncEF5mB5qqzwvJ3zEi7A

Filinich, María Isabel. (1996) Continuidad de los parques: lo continuo y lo discontinuo. Hispamérica 73, pp. 113-119.

Fortea, Carlos. (2007). La perenne continuidad de los parques: un acercamiento al maestro Julio Cortázar, en memoria suya. Vasos Comunicantes. pp. 65-68. [en línea]. Recuperado el 21 de marzo del 2010 de http://www.acett.org/documentos/vasos/vasos29.pdf

Gambetta N., Aida. (2007) Julio Cortázar, homo ludens. Relaciones (52). [en línea]. Recuperado el día 21 de marzo del 2010 de http://www.colmich.edu. mx/files/relaciones/052/pdf/Aida\%20Gambetta\%20N.pdf

Jaeck, Lois Marie. (1998) Nights of the Magician: Borges, Dreams, Cortázar, Writing and Gnosticism. Ciencia ergo sum., 5, (1). pp. 32-36.

Markus, Mario. (1990) Poemas de invierno. Madrid: Betania.

Mora Valcárcel, Carmen de. (2000). “Orientación de los gatos” (Apuntes para una poética). En breve: estudios sobre el cuento hispanoaméricano contemporáneo. pp. 99-110. [en línea]. Recuperado el 21 de marzo del 2010 en http://books. google.com.mx/books?id=cs9mSdjHV8IC\&pg=PA99\&lpg=PA99\&dq=orient acion+de+los+gatos+apuntes+para+una+poetica\&source=bl\&ots=U14w6 Wcar8\&sig=P2CzT7b8N7tUi8XCN6mxHwHfV3E\&hl=es\&ei=Q3imS5ygBYmS

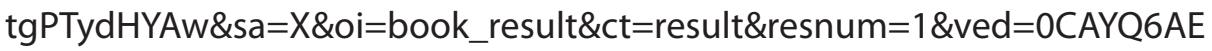
$\mathrm{wAA} \# \mathrm{v}=$ onepage $\& \mathrm{q}=\& \mathrm{f}=$ false 
Paniagua, Pablo. Literatura fractal. (2007) [en línea]. Recuperado el 28 de febrero del 2010 de http://literaturafractal.blogspot.com/

Payán Fierro, Humberto. (2009) La figura del gato en la cuentística de Julio Cortázar. [en línea]. Recuperado el 21 de marzo del 2010 de http://www.uach. mx/extension_y_difusion/synthesis/2009/08/20/La_figura_del_gato_en_ la_cuentistica_de_julio_cortazar.pdf

Pérez Cervantes, Abel. (2004) Las estrategias narrativas del doble literario en "Continuidad de los parques" de Julio Cortázar. Escritos. Revista del Centro de Ciencias del Lenguaje. (30). pp. 177-188.

Rivera Garza, Rosaura. (2009) Aquí, allá y en todas partes: los fractales. Algarabía. (63). pp. 20-25. [en línea]. Recuperado el 28 de febrero del 2010 de http:// bibliadelarazonn.blogspot.com/2010/01/revista-extractos-de-algarabia.html Ros Soriano, Maria Carmen; Polo García, Victorino. (1990) El caracter de la literatura desmontable y reutilizable en los cuentos de Cortázar. Anales de filología hispánica, pp. 243-253. [en línea] Recuperado el 21 de marzo del 2010 de http://revistas.um.es/analesfh/article/viewFile/57861/55741

Simone, Raffaele. (2001) Fundamentos de lingüística. (2a. ed). España: Ed. Ariel. Soto Calzado, Inocente. (2009) El ídolo de las cícladas y Julio Cortázar. Recuperado el 21 de marzo del 2010 de http://www.fe.ccoo.es/andalucia/docu/p5sd5369.pdf

Talanquer, Vicente. (2009) Fractus, fracta, fractal: fractales, de laberintos y espejos. (1a. reimp. de la 3a. ed.). México: FCE.

Viñuela, Alberto. (2001) Recursividad en la literatura. [en línea]. Recuperado el día 28 de febrero del 2010 en http://www.arrakis.es/ sysufus/litfr.html

Zavala Lauro. (2006) Una conclusión para empezar: fragmentos, detalles y fractales. El ojo en el caleidoscopio. México: UNAM.

,(2004) Cartografías del cuento y la minificción. (1a. ed.). Sevilla: Renacimiento. (1993) Humor, Ironía y Lectura. Las Fronteras de la escritura literaria. México: UAM. - De la teoría de la novela a la teoría de la minificción. Ponencia magistral Segundo Encuentro de Minificción en el Aula Magna de la Facultad de la UNAM. 